\title{
Reproducing the Proximal Femur Anatomy: Modular Femoral Component
}

\author{
Aldo Toni, Francesco Castagnini, \\ and Susanna Stea
}

\subsection{Neck Modularity}

Stem modularity can be classified according to the coupling location: distal, mid-stem, and proximal [1]. Mid-stem and proximal modularity have been more frequently used. Either the junction is located proximal or distal (mid-stem) to the neck osteotomy (Fig. 8.1). Proximal modularity with modular necks was introduced in 1987 by Cremascoli Ortho (Milan, Italy), in order to provide independent combinations of version, offset, and length [1].

Rationale of modular necks. The rationale for proximal modularity with modular necks was the achievement of a better soft tissue balancing and was to reduce the occurrence of prosthetic impingement $[1,2]$. The best candidates for neck modularity, where the most remarkable benefits are obtained, are subjects with unconventional hip anatomies and biomechanics $[1,2]$. In these cases, standard acetabular and femoral compo-

A. Toni $(\bowtie)$

Casa di Cura Madre Fortunata Toniolo,

Bologna, Italy

F. Castagnini

Ortopedia-Traumatologia e Chirurgia protesica e dei reimpianti d'anca e di ginocchio, IRCCS Istituto

Ortopedico Rizzoli, Bologna, Italy

\section{S. Stea}

Laboratorio di Tecnologia Medica, IRCCS Istituto

Ortopedico Rizzoli, Bologna, Italy

e-mail: stea@tecno.ior.it nent orientation adjustment to achieve adequate reciprocal positioning may be ineffective due to the limited bone fit and coverage. Such suboptimal implant orientation may result in restricted range of motion, abductor dysfunction, and increased risks of dislocations and other impingement-related events $[1,3]$. The bony and soft tissue alterations in hip dysplasia are a clear example (Fig. 8.2) [1, 2]. The short anteverted neck and the inadequate abductor muscles may be correctly reconstructed using modular components, independently tuning the soft tissue tension and the leg length. Moreover, modularity may provide adequate correction of the combined version. Similar considerations can be made for coxa vara: a conventional implant, with progressive neck lengths according to implant size, may restore the offset using a larger size, but sometimes at the cost of an unacceptable leg length discrepancy (Fig. 8.3) [1-3]. Modularity may also be beneficial in post-traumatic cases, where the abnormal acetabular or femoral morphologies may influence the component position. In revision cases, when the acetabular bone loss may compromise the socket positioning, modularity may improve joint stability and reduce impingement, compensating imperfect acetabular implant orientation and/or soft tissue tension [1]. Even in case of standard primary arthroplasties looking relatively simple, as the proximal femoral anatomy significantly varies between patients and is not that predictable [2], proximal modularity 
Fig. 8.1 Stem modularity can be classified according to the junction location: on the left mid-stem modularity (junction distal to the neck osteotomy) and on the right proximal femoral modularity (junction proximal to the neck osteotomy)
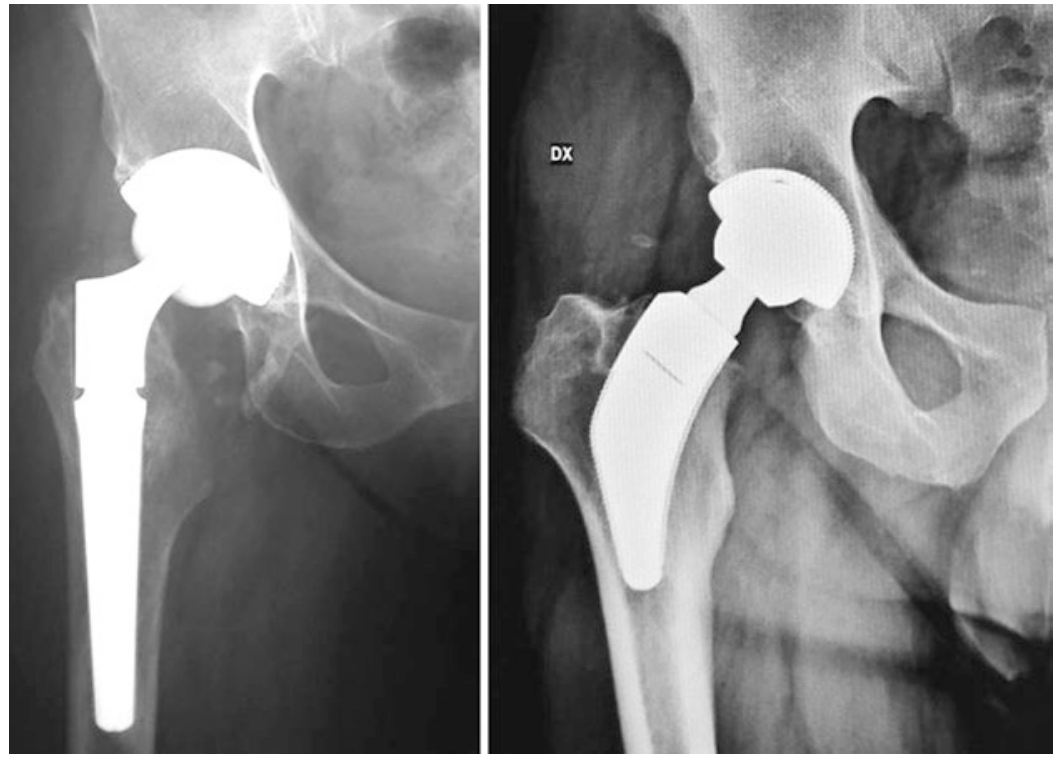

with modular necks may be of clinical interest. Males usually have long necks with higher neckshaft angles and low anteversion; on the contrary, females show short, varus, and anteverted necks [2]. Most stem designs have size-proportional neck lengths, affecting leg length and offset, which may address partially this wide variability [2]. It was estimated that tapered stems with metaphyseal fit designs require at least 15 sizes distributed in three metaphyseal configurations, and two different neck-shaft angles to match the frontal anatomy of $85 \%$ of the femurs [4]. Moreover, uncemented stems often have limited version freedom during implantation. Thus, proximal modularity with modular necks has theoretical advantages in outlier anatomies as well as in standard cases, more closely matching the native proximal femur anatomy than conventional stems. Due to these reasons, we frequently used in our institution the modular neck implants in cases of conventional and, mostly, unconventional hip anatomies.

Clinical results of modular necks. There is solid evidence that shows the reliability of welldesigned modular neck implants regarding their capacity to restore the native proximal femoral anatomy and to provide good long-term clinical outcomes. Montalti et al. [5] reported good anatomical reconstructions in severe dysplastic hips using modular necks $\left(\mathrm{AncaFit}^{\circledR}\right.$ stem, Cremascoli
Ortho, Milan, Italy). In particular, the use of a high prosthetic hip center reconstruction, combined with modular necks, improved the biomechanics and the offset restoration, with good to excellent clinical results and only a case of cup aseptic loosening after a minimum follow-up of 10 years. Archibeck et al. [6] reported a comparison between 100 primary total hip arthroplasties (THAs) with modular neck stem design and 100 primary THAs without (respectively Kinectiv $^{\circledR}$ and M/L Taper ${ }^{\circledR}$ stems, ZimmerBiomet, Warsaw, USA). The offset and leg length were restored to within $1 \mathrm{~mm}$ in $85 \%$ of the modular hips and in $60 \%$ of the monoblock stems. On the contrary, Duwelius et al. [7] failed to demonstrate superior clinical outcomes at 2-years follow-up in a similar comparison involving the same implants. Nevertheless, a better reconstruction of leg length and offset characterized the modular neck cohort.

Our experience: The regional arthroplasty registry of Emilia Romagna. In the regional arthroplasty registry of Emilia Romagna, the 15-year cumulative implant survival rates were found to be similar between 16,575 modular implants (557 being exposed to risk at 15 years) and 35,620 monoblock implants (1781 being exposed to risk at 15 years) performed for primary osteoarthritis, at $90.8 \%$ and $91 \%$, respectively [8]. The rates of aseptic loosening were 

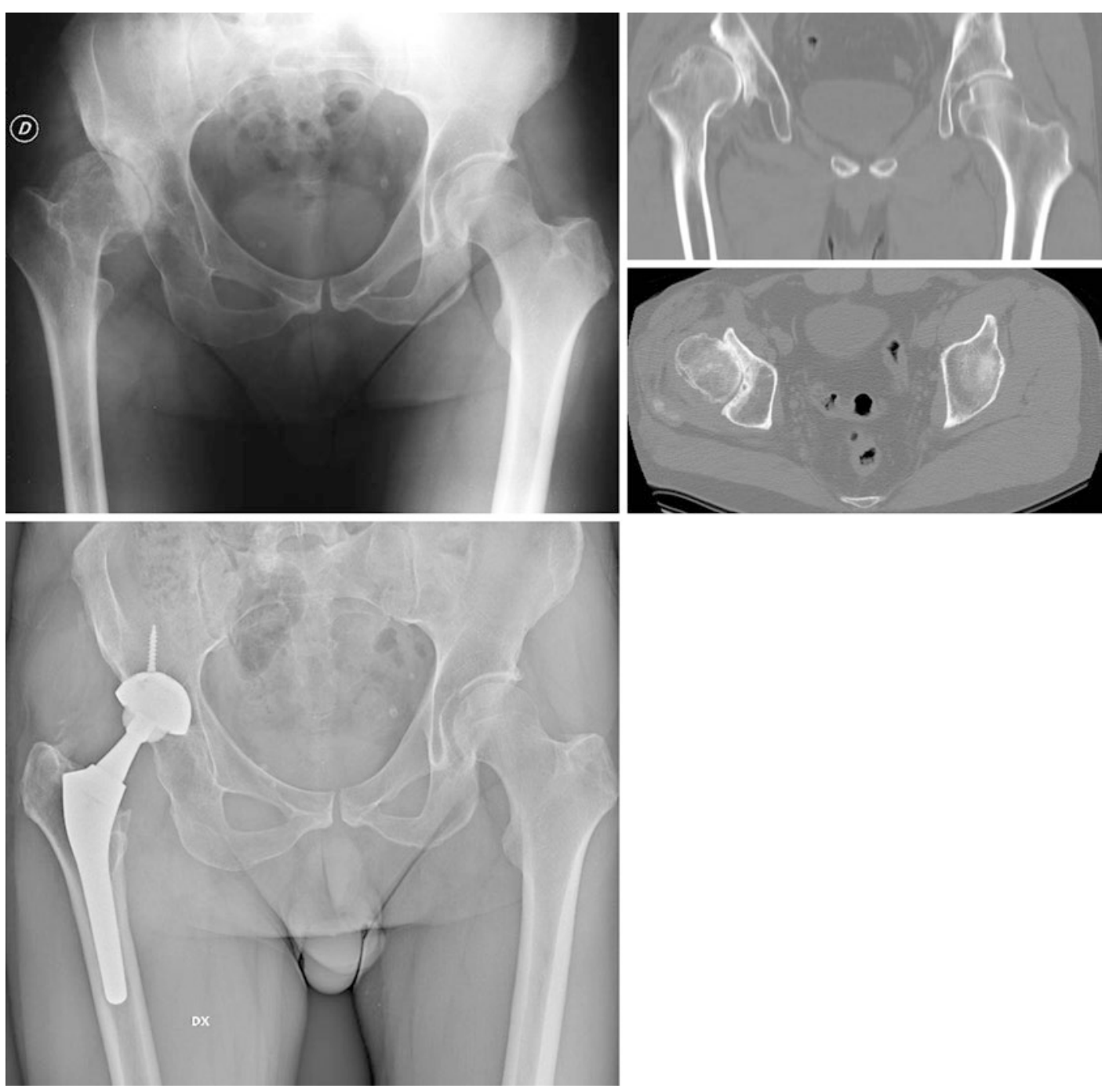

Fig. 8.2 A Crowe III dysplasia in a 45-year-old male was treated with a $\mathrm{Ti}$ modular neck implant (Ancafit, Cremascoli Ortho, Milan, Italy): the use of a long neck

allowed for the proper restoration of leg length and offset, achieving an excellent result at 13 years

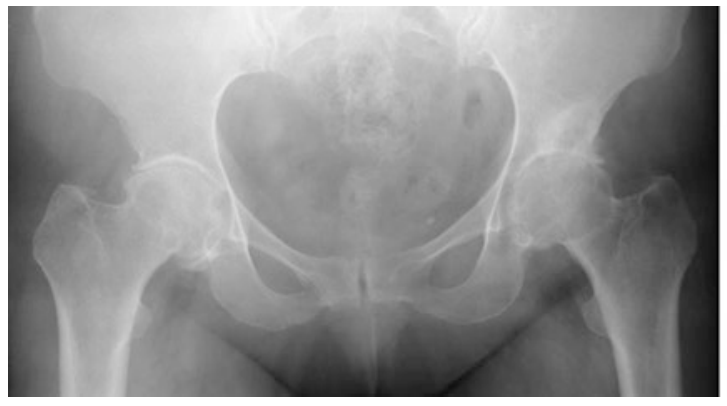

Fig. 8.3 Modular implants are particularly useful in outlier anatomies, like coxa vara. A varus, retroverted neck (Apta, Adler Ortho, Milan, Italy) was used to restore the

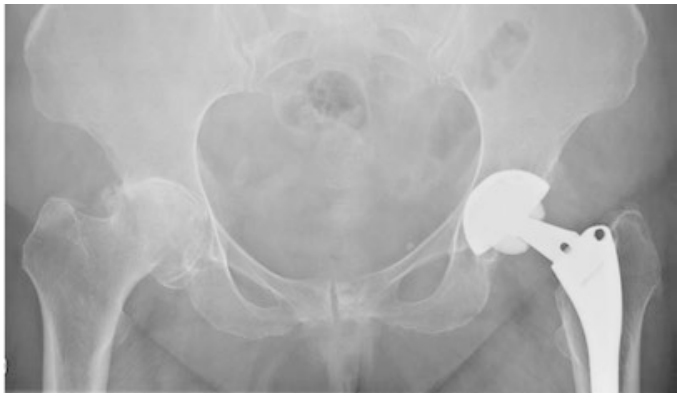

proper neck-shaft angle, offset, and leg length, achieving a satisfying result at 8 years 
inferior in the modular group $(0.4 \%$ vs. $0.7 \%$ for isolated acetabular implant loosening; $0.6 \%$ vs. $0.8 \%$ for isolated femoral stem loosening; and $0.1 \%$ vs. $0.4 \%$ for loosening of both the components), as well as the rate of revision due to polyethylene wear $(0.04 \%, 7$ implants out of 16,575 THAs), but none of those differences reached statistical significance. In terms of revisions due to prosthetic instability, no differences could be detected between the two groups. These data may suggest that modular necks allow for a better component interaction, enabling to reduce the mechanical stresses on component fixation and preserving them from aseptic loosening. Considering only the THAs performed due to congenital pathologies (e.g., developmental hip dysplasia), the results between the two groups were more striking. The modular neck implants (2805 cases with 238 being exposed to risk at 15 years) achieved a survival rate of $93.3 \%$ at 15 years, whereas conventional implants (3707 cases with 389 being exposed to risk at 15 years) had a lower performance with $89.6 \%$ of survivorship. Regarding the reasons for revision, the two groups had similar revision rates for early prosthetic instability (within the first 3 months), but modular neck THAs had an inferior revision rate for recurrent dislocations $(0.5 \%$ vs. $0.8 \%)$. Revisions for aseptic cup loosening were significantly lower with modular necks $(0.5 \%$ vs. $1.9 \%)$. This finding showed that, in the modular neck cohort, the revision rate for aseptic cup loosening was comparable to THAs implanted for primary osteoarthritis and was four times lower than conventional implants used for congenital pathologies. On the other hand, in the cohort of congenital pathologies, a rate of $0.5 \%$ of neck fractures was reported for the modular neck implants.

Insight into modular neck failure. Due to the frequent excessive corrosion at the modular neck-stem junction and the related clinical complications (e.g., neck breakage, adverse local tissue reaction to metal debris), the routine use of modular neck implants has been frequently questioned [1, 9]. In a recent study by Graves et al. [9] that describes the outcomes of the Australian registry, the revision rate for all reasons at 10 years was $9.7 \%$ in modular necks, whereas $5.1 \%$ for the conventional stem. However, when the modular neck cohort was split into chromium-cobalt (Cr-Co) necks and Titanium (Ti) necks, the latter performed better: at 10 years, $7.4 \%$ of the $\mathrm{Ti}$ implants were revised, suggesting that the neck alloy is an important predictive factor. The first modular necks were fabricated in Ti alloy, with a taper connection mated to a Ti alloy stem [1]. Of the reported breakage and dissociation of the components, neck failures were mostly due to insufficient fatigue strength (Fig. 8.4) [1, 9]. Thus, Cr-Co alloy necks were proposed as a stronger alloy alternative and implanted on $\mathrm{Ti}$

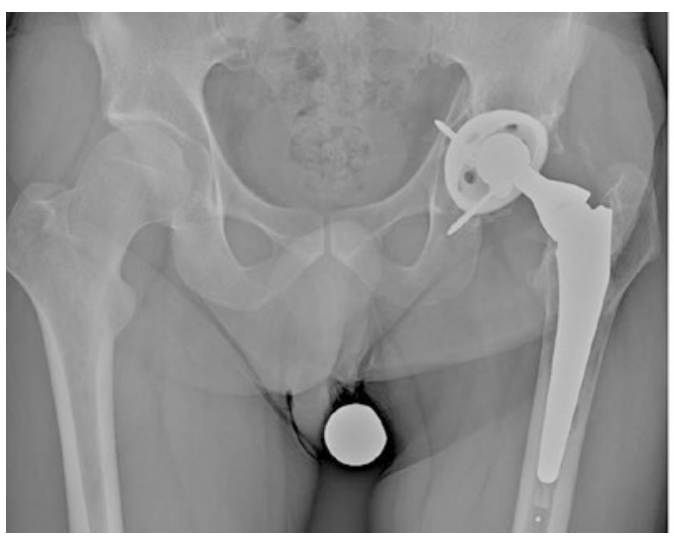

Fig. 8.4 Ti proximal modularity may face disassembling (left image) and neck failure (right image): disassembling occurred after a trauma 20 years after the implantation.

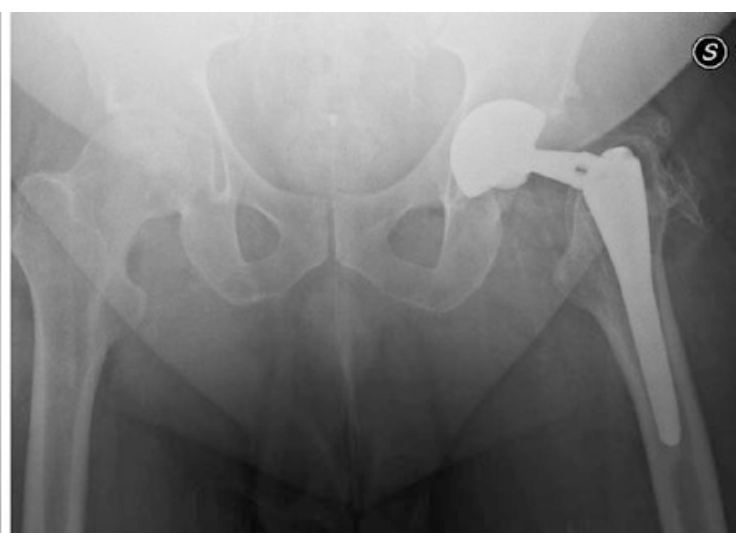

The fracture occurred in severe obesity $(150 \mathrm{~kg}$, 65-yearold male) 
alloy stems in order to prevent fractures $[1,10$, 11]. The new $\mathrm{Cr}-\mathrm{Co}$ necks experienced lower rates of fractures (to date, no implant breakage in the Australian registry) [9]. However, excessive corrosion at the taper junction, primarily related to abnormal micromotions at its site (mechanically assisted crevice corrosion), emerged as a devastating complication [1, 10-15]. A famous example may be given by the 2012 recall of the Rejuvenate devices (Stryker, Mahwah, USA) where $\mathrm{Cr}-\mathrm{Co}$ necks were mated to Ti alloy stems. De Martino et al. [10] analyzed 60 Rejuvenate stems that were removed for multiple reasons; the totality of the retrievals showed severe signs of fretting corrosion at the neck-stem modular taper junction, starting soon after the implantation (less than 4 weeks) and increasing over the time. In contrast, the head-neck tapers only showed negligible corrosion signs. The authors suggested that the neck-stem junction was subject to a cantilever bending: the medial and lateral sides of the neck were cyclically compressed against the correspondent part of the stem, describing a small amplitude oscillatory motion [10]. Nawabi et al. [11] described the results of 216 Rejuvenate THAs, highlighting necrosis and adverse local tissue reactions similar to metal-onmetal bearing prostheses as a consequence of the cantilever bending. The source of metal ions was the mechanically assisted crevice corrosion: the fluid entering the modular junction repassivated the titanium alloy, causing acid release and $\mathrm{Ti}$ or Co dissolution [10-15]. Due to these severe problems, the American Association of Hip and Knee Surgeons elaborated a specific algorithm for risk stratification, designing $\mathrm{Cr}-\mathrm{Co}$ neck implants at moderate or severe risk [12]. A strict follow-up including standard radiographs, periodical metal ion level tests, and cross-sectional images (MARS-MRI or CT scan) was suggested, in order to establish if a revision surgery was recommended. On the other hand, when Ti necks were compared to $\mathrm{Cr}-\mathrm{Co}$ necks, less degradation was evident and ion release was modest and within a noncritical range [13-15]. A study by Kop et al. [13] suggested that Ti necks are more corrosion resistant and frequently subject to cold welding. The cold welding at the neck-stem modular junction was probably beneficial in terms of reducing fretting corrosion but rendered disassembling a troublesome (impossible) procedure in $22 \%$ of the cases. Despite the safer $\mathrm{Ti}$ neck profile, a few catastrophic events related to neck breakage should not be forgotten $(0.2 \%$ in the Australian registry) [9]. It is not easy to delineate the reasons for Ti neck breakage: the implant design plays a capital role, as few models have been frequently involved [13-15]. Moreover, retrieval studies on Ti neck breakage found that varus high-offset modular necks were at higher risk of fracture when implanted in young, active, overweight patients [1, 13-15].

\subsection{Head Modularity}

Similarly to modular necks, modular heads were introduced in the 1980s, aiming to restore better prosthetic hip biomechanics [16]. The success was outstanding and, in the 1990 s, $90 \%$ of the implants had a head-neck modularity [16]. Nowadays, head modularity is a capital element in THA as it allows the surgeon to use different bearing surfaces, to more accurately restore offset and leg length, to improve stability, and to facilitate revision procedures [16-18]. Usually, head modularity occurs at a Morse taper, resulting in a force-fit connection (taper locking) that resists the axial and torsional forces [16]. Unfortunately, there is no standard taper. Tapers are fabricated with different configurations and angles, with several variations among manufacturers and hip devices [16-18]. Thus, the surgeons must carefully assess the compatibility between the new head and the well-fixed stem in case of partial revisions [16-18]. Although the benefits related to head modularity remarkably outweigh the cons, few drawbacks should be noticed: disassembling and excessive corrosion [17]. Dissociation of the head is anecdotic in the modern implants, usually occurring after trauma and secondary to mismatches [17]. Severe and clinically troublesome corrosion almost uniquely occurs with large metal heads, being ceramic balls involved in very few cases. The taper is subjected to mechanically assisted crevice corro- 
sion due to oxidation and micromotion, similarly to the neck-stem junctions that are usually Morse tapers too [17]. The main factors increasing corrosion at the head-neck interface occur with different metal combinations, larger heads (>32 mm), shorter tapers, high head offset (e.g., XL head), and active and/or obese patients [17]. Despite those few concerns, the routine use of head-neck modularity in primary THAs is not challenged. Furthermore, the use of modular head-neck adapter systems seems particularly important in revision settings [18]. Such systems allow the reduction of leg length discrepancy and the loss of offset, improving the biomechanics and the stability of the revised prosthetic hip. In a retrospective series including 95 patients, Hoberg et al. [18] described 95 revisions requiring the use of BioBall ${ }^{\circledR}$ system (Merete, Berlin, Germany). The survival rate was $92.8 \%$ at 8 years, two patients requiring a further procedure due to recurrent dislocations. No corrosion was noticed in the revised cases.

\subsection{Femoral Component Modularity Contributes to Personalizing the Hip Reconstruction}

Proximal femoral modularity is a useful tool to optimize prosthetic hip biomechanics and to potentially reduce complications related to poor component interaction (edge loading, prosthetic impingement, and related complications such as instability). Each patient has a unique hip biomechanics that may even vary with age. Three capital parameters of hip biomechanics, femoral offset and hip rotation center and combined anteversion of femur and acetabulum, are randomly and independently located even in conventional hip morphologies [1-4]. Restoring the native hip anatomy when performing hip replacement is a sound option for optimizing prosthetic function and biomechanics, and overall clinical outcomes and patients' satisfaction. However, as conventional implants, even with modular heads, may only address a few hip anatomies, most of the patients have their native hip anatomy altered after reconstruction. Thanks to the independent tuning of lengths and angles in the three planes, regardless the stem size, proximal modularity (head and neck) may effectively reproduce the constitutional hip biomechanics within millimeters $[1,2]$. Thus, the muscle lever arms can be finely reconstructed and the combined anteversion optimized. A wider articular excursion, a more uniform distribution of joint forces, and an improved component interaction consequently result, highlighting that personalizing hip replacement using modularity is not without tangible outcomes [4]. Proximal femoral modularity is even more desirable in case of outlier anatomies, when conventional implants with minimal modularity (head) grossly fail to reconstruct the hip biomechanics [1, 2, 5].

\subsection{Modularity: Guidelines for Users}

Complex cases with unusual anatomies and biomechanics, like dysplastic hips, may be the best candidates for modular implants $[2,5]$. Up to now, well-designed femoral head/neck modularity has proven to be reliable, achieving positive long-term results in such cases (Fig. 8.5) [1, 5]. On the contrary, a regular use of modular neck implants has been universally discouraged [9]. Excluding its costs (15-25\% more expensive than conventional implants), adding a modular junction carries some additional risks related to corrosion, disassembling, and modular implant failure [1]. Lessons learned with modular implant failures helped us to draw important recommendations and restrictions [16]. Mixing alloys should be avoided due to corrosion and ion release. $\mathrm{Cr}-$ Co heads on Ti necks can be admitted; on the contrary, $\mathrm{Cr}-\mathrm{Co}$ necks in Ti stem junctions should be avoided [1,9-13]. Every taper is designed to better resist torsional loading rather than bending one [16]. Thus, in young, active, and overweight patients, modular head and neck options that provide high femoral offset should be adopted with care, or better, discouraged [13-15]. In these cases, modular junction corrosion and Ti neck fatigue fractures are more likely to occur [13-15]. 

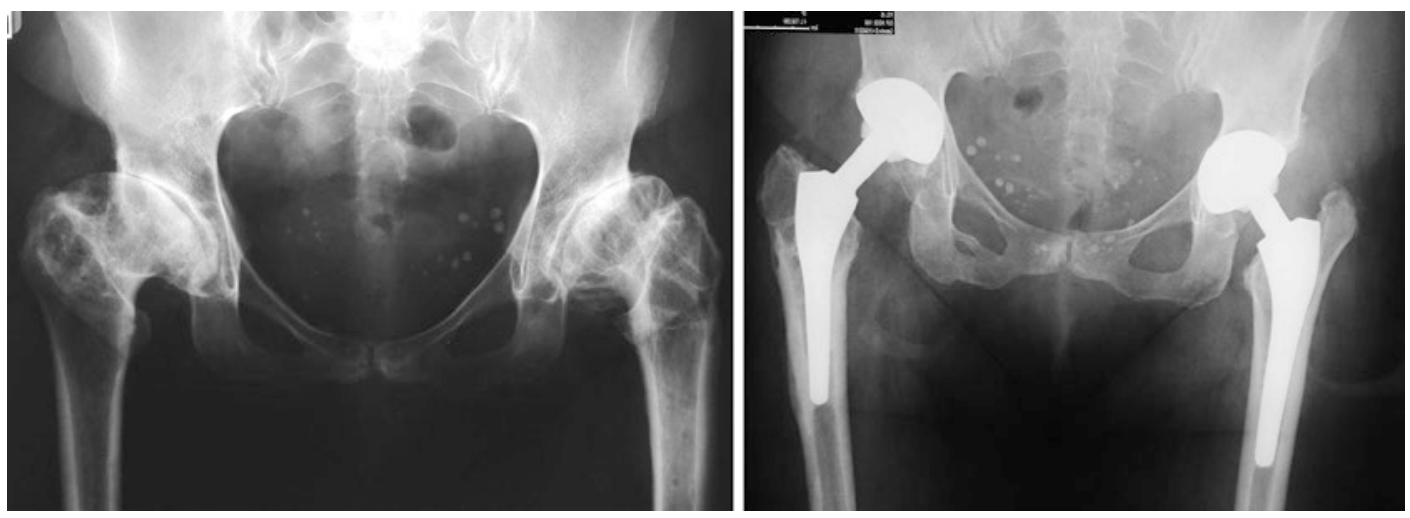

Fig. 8.5 Modular necks are useful implants in case of difficult anatomies. A case of dysplasia was treated with modular THAs (Ancafit, Cremascoli Ortho, Milan, Italy), achieving a good radiographic result after 15 years

Thus, problems with taper can be avoided reducing the bending load at taper interface and, in case of modular heads, increasing the taper strength acting on diameter and length [13-15]. It is important to highlight that a proper assembly is capital: no third body should be entrapped in the taper connections [9-17]. A careful surgical technique is universally recommended to avoid taper failures [9-17]. Although modularity was proven reliable when handled with the abovementioned recommendations, new developments should be welcomed, aiming to produce safer modular junctions. The microstructure and the grain size of the Ti alloy were advocated as important factors: in particular, as most of the cracks initiated and propagated between two alfa-lamellae, avoiding or reducing such elements might be beneficial [14]. Such a development should be accompanied by a proper implant design, a factor significantly affecting the long-term results [13-15]. Up to now, a 100\% safe design has not been found. The modular junction design of Ancafit ${ }^{\circledR}$ implants proved good [8]: only 2 neck breakages out of 3148 cases occurred at a minimum follow-up of 5 years. The modest offset range $(13.5 \mathrm{~mm})$ may have probably played an important role in such a success. Another positive experience was the Modula $^{\circledR}$ system (Adler Ortho, Milan, Italy), which is available for different stem designs. This modular Ti neck system provides independent tridimensional tuning of three parameters (length, offset, version) achieving 27 combinations per side and an offset range of $26 \mathrm{~mm}$ [19]. Although initial data showed an excessive rate of neck fractures, in particular in young patients and highoffset implants (unpublished results), the more recent use of a second generation "reinforced" neck system has generated a significant improvement in the fracture rate. To date, at the last follow-up (December 2015), no fracture occurred with the second-generation system in 1689 implants at a mean follow-up of 1.8 years (range: 0-3.7) [8].

\section{Clinical Case}

A 64-year-old female patient came presented to our clinic after a long history of right groin pain. When she was a child, the patient was conservatively treated for congenital hip dysplasia, achieving modest results. The patient had a residual limping, requiring the regular use of a walking cane and a severe lower limb discrepancy (Harris hip score: 23.8 points).

The anteroposterior X-rays demonstrated a bilateral Crowe III dysplasia (Fig. 8.6). In both hips, the articular degeneration was severe and the greater trochanters were very deformed. The right lower limb was $2 \mathrm{~cm}$ shorter. The computed tomography (CT) scan showed a dysplastic, small and shallow acetabulum, and a remarkable neck antetorsion $\left(27^{\circ}\right)$. The gluteal muscles were short and hypotrophic. 

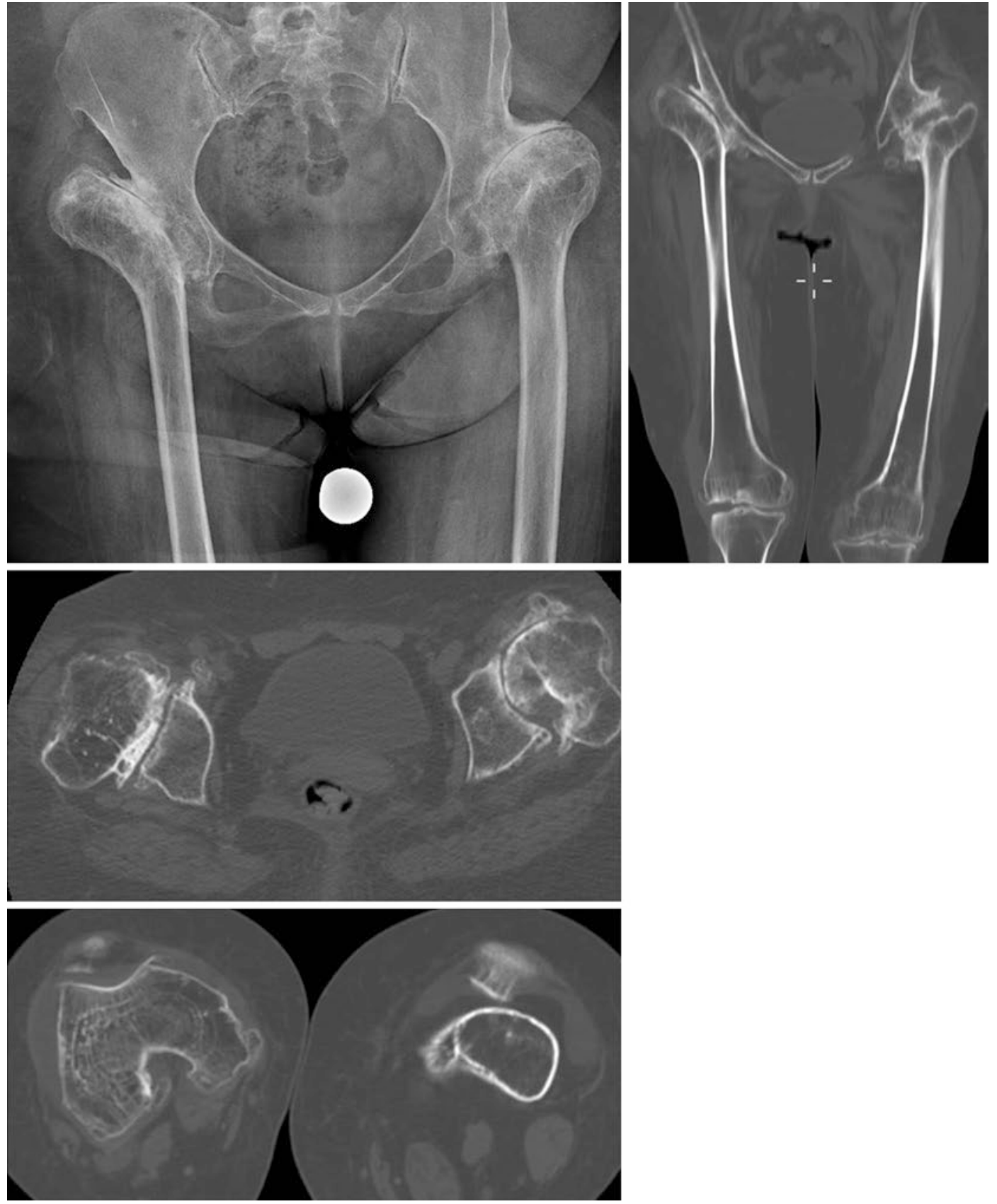

Fig. 8.6 (clinical case) The anteroposterior radiograph and CT scan showed bilateral dysplastic hips, severely shallow acetabula, and marked femoral antetorsion, with deformed trochanters, leg length discrepancy, small and minimum femoral offsets and abductor deficiencies

A right cementless total hip arthroplasty was performed, using an anterolateral approach. The cup was a highly porous titanium socket (TiPor, Adler Ortho, Milan, Italy), positioned in a high hip center. A modular tapered stem was implanted (Acuta, Adler Ortho, Milan, Italy). The shortest varus titanium (Ti) neck was positioned in order to restore the offset without damaging the weak abductors. The stem antetorsion was controlled, using the tapered stem-retro- 
verted modular necks were not necessary. Delta ceramic bearing surfaces were chosen with a $32 \mathrm{~mm}$ ball (CeramTec, Plochingen, Germany). The greater trochanter was modeled and the abductors were sutured and re-tensioned. The implant proved stable and a good range of motion was achieved, with a residual $0.8-\mathrm{cm}$ lower limb discrepancy to avoid excessive stresses on the gluteal muscles.

After 5 years, the patient was satisfied with the final result: the Harris hip score was 85.8 points. The gluteal muscles were still hypotrophic and a slight limping was evident: a walking cane was required only for long distances. On the radiographs, the implant showed good osseointegration (Fig. 8.7).

Severe dysplastic cases should be treated with specific modular implants. The use of highly porous cups and ceramic-on-ceramic couplings reduce the rate of wear and aseptic loosening, even in case of abnormally high joint reaction forces. Tapered stems may efficaciously control the combined anteversion, mostly when the stem antetorsion is higher than $25^{\circ}$. Modular necks are useful to independently fine-tune the offset, the length, and the version, restoring a good abductor lever arm and physiological softtissue tension.

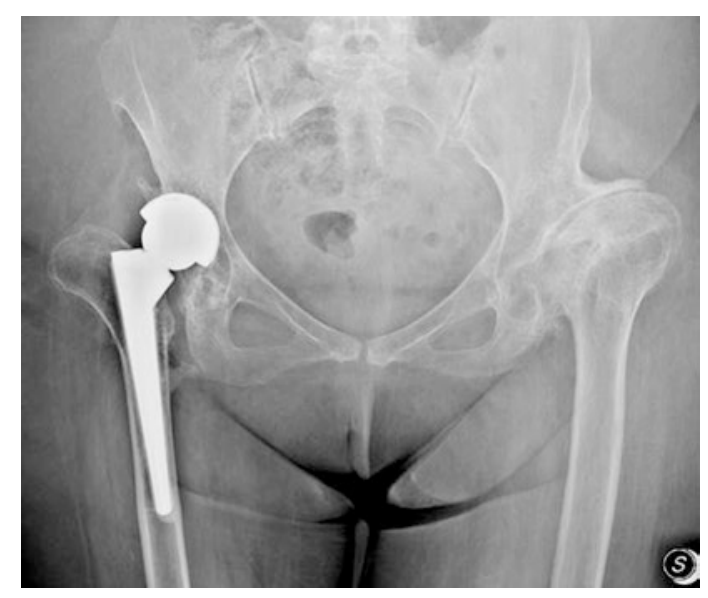

Fig. 8.7 (clinical case) After 5 years, the anteroposterior radiograph showed a very good osseointegration of the components and the restoration of a proper offset, avoiding excessive elongation of the gluteal muscles. In this case, proximal modularity was necessary to manage a very complex hip anatomy

\section{References}

1. Srinivasan A, Jung E, Levine BR. Modularity of the femoral component in total hip arthroplasty. J Am Acad Orthop Surg. 2012;20(4):214-22.

2. Traina F, De Clerico M, Biondi F, Pilla F, Tassinari E, Toni A. Sex differences in hip morphology: is stem modularity effective for total hip replacement? J Bone Joint Surg Am. 2009;91(Suppl 6):121-8.

3. Matsushita A, Nakashima Y, Fujii M, Sato T, Iwamoto Y. Modular necks improve the range of hip motion in cases with excessively anteverted or retroverted femurs in THA. Clin Orthop Relat Res. 2010;468(12):3342-7.

4. Massin P, Geais L, Astoin E, Simondi M, Lavaste F. The anatomic basis for the concept of lateralized femoral stems: a frontal plane radiographic study of the proximal femur. J Arthroplast. 2000;15(1): 93-101.

5. Montalti M, Castagnini F, Giardina F, Tassinari E, Biondi F, Toni A. Cementless total hip arthroplasty in Crowe III and IV dysplasia: high hip center and modular necks. J Arthroplast. 2018;33(6):1813-9.

6. Archibeck MJ, Cummins T, Carothers J, Junick DW, White RE Jr. A comparison of two implant systems in restoration of hip geometry in arthroplasty. Clin Orthop Relat Res. 2011;469(2):443-6.

7. Duwelius PJ, Burkhart B, Carnahan C, Branam G, Ko LM, Wu Y, Froemke C, Wang L, Grunkemeier G. Modular versus nonmodular neck femoral implants in primary total hip arthroplasty: which is better? Clin Orthop Relat Res. 2014;472(4):1240-5.

8. Registro dell'implantologia protesica ortopedica RIPO. https://ripo.cineca.it/. Accessed 25 May 2018.

9. Graves SE, de Steiger R, Davidson D, Donnelly W, Rainbird S, Lorimer MF, Cashman KS, Vial RJ. The use of femoral stems with exchangeable necks in primary total hip arthroplasty increases the rate of revision. Bone Joint J. 2017;99-B(6):766-73.

10. De Martino I, Assini JB, Elpers ME, Wright TM, Westrich GH. Corrosion and fretting of a modular hip system: a retrieval analysis of 60 rejuvenate stems. J Arthroplast. 2015;30(8):1470-5.

11. Nawabi DH, Do HT, Ruel A, Lurie B, Elpers ME, Wright T, Potter HG, Westrich GH. Comprehensive analysis of a recalled modular total hip system and recommendations for management. J Bone Joint Surg Am. 2016;98(1):40-7.

12. Kwon YM, Fehring TK, Lombardi AV, Barnes CL, Cabanela ME, Jacobs JJ. Risk stratification algorithm for management of patients with dual modular taper total hip arthroplasty: consensus statement of the American Association of hip and knee surgeons, the American Academy of orthopaedic surgeons and the hip society. J Arthroplast. 2014;29(11):2060-4.

13. Kop AM, Keogh C, Swarts E. Proximal component modularity in THA--at what cost? An implant retrieval study. Clin Orthop Relat Res. 2012;470(7): 1885-94. 
14. Fokter SK, Rudolf R, Moličnik A. Titanium alloy femoral neck fracture--clinical and metallurgical analysis in 6 cases. Acta Orthop. 2016;87(2):197-202.

15. Kretzer JP, Jakubowitz E, Krachler M, Thomsen M, Heisel C. Metal release and corrosion effects of modular neck total hip arthroplasty. Int Orthop. 2009;33(6):1531-6.

16. Morlock M. Modularity in orthopaedics. J Traum Orthopae. 2017;5(3):60-3.

17. Wight CM, Lanting B, Schemitsch EH. Evidence based recommendations for reducing head-neck taper connection fretting corrosion in hip replacement prostheses. Hip Int. 2017;27(6):523-31.
18. Hoberg M, Konrads C, Huber S, Reppenhagen S, Walcher M, Steinert A, Barthel T, Rudert M. Outcome of a modular head-neck adapter system in revision hip arthroplasty. Arch Orthop Trauma Surg. 2015;135(10):1469-74.

19. Ollivier M, Parratte S, Galland A, Lunebourg A, Flecher X, Argenson JN. Titanium-titanium modular neck for primary THA. Result of a prospective series of 170 cemented THA with a minimum follow-up of 5 years. Orthop Traumatol Surg Res. 2015;101(2):137-42.

Open Access This chapter is licensed under the terms of the Creative Commons Attribution 4.0 International License (http://creativecommons.org/licenses/by/4.0/), which permits use, sharing, adaptation, distribution and reproduction in any medium or format, as long as you give appropriate credit to the original author(s) and the source, provide a link to the Creative Commons license and indicate if changes were made.

The images or other third party material in this chapter are included in the chapter's Creative Commons license, unless indicated otherwise in a credit line to the material. If material is not included in the chapter's Creative Commons license and your intended use is not permitted by statutory regulation or exceeds the permitted use, you will need to obtain permission directly from the copyright holder. 